\title{
The Impact of Growth in Height on the Physical Competencies of Children
}

\author{
Mathieu F. McKinnon, Angela M. Kolen* \\ Human Kinetics, St. Francis Xavier University, Antigonish, Canada \\ *Corresponding author: akolen@stfx.ca
}

\begin{abstract}
It is important to recognize or control for the impact of physical growth to evaluate changes in children's physical performance as they get older particularly when involved in an exercise or training program. This study examined the effect of growth in height on four physical competency tasks in children. Data were collected twice from 206 children, aged 8 to 11 years at first data collection. These participants completed the Progressive Aerobic Cardiorespiratory Endurance Run [PACER], a timed plank, left- and right-hand grip strength, and had their height and mass measured in consecutive years. Growth in height was used in dimensional analyses to predict the participants' expected improvement in their physical competency measures. The expected improvement in PACER, timed plank and left- and right-hand grip strength was compared to actual differences in the children's performance using repeated measures t-tests. Significantly greater improvements than expected were found for left $(t(167)=-4.731$, $p=0.000)$ and right-hand grip $(t(168)=-4.579, p=0.000)$. Actual performance was significantly less than expected for the timed plank $(t(172)=-2.743, p=0.003)$. No significant differences in actual versus expected performance were found for the PACER $(t(164)=-1.023, p=0.154)$. Although children's growth in height accounted for a proportion of their performance, it was inconsistent suggesting other biologic and non-biologic factors also impact their physical competencies over time.
\end{abstract}

Keywords: dimensional analysis, grip strength, plank, PACER

Cite This Article: Mathieu F. McKinnon, and Angela M. Kolen, "The Impact of Growth in Height on the Physical Competencies of Children." Journal of Physical Activity Research, vol. 3, no. 1 (2018): 6-10. doi: 10.12691/jpar-3-1-2.

\section{Introduction}

Growth refers to the increase in size of the body as a whole and of its individual parts [9]. Humans follow a general growth pattern with relatively rapid growth during infancy and early childhood, consistent, steady growth during middle and late childhood, followed by another period of rapid growth during adolescence, and finally cessation of growth when adulthood is reached [13]. Although height and weight follow this general growth curve, it should be kept in mind that factors other than growth have the potential to influence weight in a growing child. These factors include over and undernutrition as well as participation in physical activity, exercise, and sport. The growth of height, weight, other bodily systems, and structures are primarily a result of the release of hormones such as human growth hormone and testosterone [12]. Given that Lynch et al. [8] demonstrated that the total amount of muscle was the main determinant in the force generating capacity of muscle, it is reasonable to assume that improvements in physical capabilities with age are due then primarily to increases in muscle size as a result of the normal physical growth of children. It is also reasonable to assume that as a child grows, his or her nervous system also grows to allow for more precise control of muscle. Caroni and Grandes [3] found in mice insulin-like growth factors that promoted nerve sprouting in skeletal muscle. These findings potentially support the idea that as a child grows the number of motor neurons in skeletal muscle increases and thereby may allow for greater muscular control [5], and consequently an advantage in completing physical competency tasks. Given the possible impact of the normal process of growth on the physical skills and physical fitness of children, the purpose of this study was to determine whether increases in the physical competencies of children over time could be explained primarily by commensurate increases in their height, a measurement of their growth.

\section{Methods}

\subsection{Participants}

Data examined in this study were obtained from a larger research project; the Canadian Assessment of Physical Literacy (CAPL) [7]. The purpose of the CAPL was to determine baseline physical literacy scores for children ages 8 to 12 years across Canada. From one data collection site in a rural community, repeat measurements were obtained from 206 children for the Progressive Aerobic Cardiovascular Endurance Run (PACER), timed plank, right- and left-hand grip strength, height, and mass. Prior to each data collection session, the parents/guardians 
of the children provided signed informed consent and the children themselves provided verbal assent. A child's refusal of assent overrode parental/guardian consent. The parents/guardians also completed a health screening form for their child to identify health issues and/or limitations that might impact their child's ability for the physical activities in this study. Ethical approval for CAPL was obtained nationally, as well as regionally from the local university and school board from which the children were recruited.

\subsection{Procedures}

Data collectors instructed the children to give maximal effort in each measure of physical competency. The timed plank and right- and left-hand grip strength were obtained from the children in a one-on-one format with each child working with a data collector when it was their turn to perform the task. The PACER was collected in a group setting with several data collectors. All data were collected from the children at their school, during the school day.

The PACER, a maximal graded exercise test, was used to assess cardiorespiratory endurance [12]. The children were asked to run between two pylons, set 20 meters apart, at a cadence that increased when each stage was completed. Each child ran between the pylons until unable to keep the pace. Data collectors monitored laps; one fault was allowed and the run terminated when the child could not reach the pylon a second time within the set cadence. The number of completed laps were recorded for each child.

Right- and left-hand grip strength provided an assessment of upper body strength and was measured using a handgrip dynamometer (Smedley Grip Tester, Gopher, Toronto, ON, Canada) following the protocol of the Canadian Society for Exercise Physiology (CSEP) [4]. The handgrip dynamometer was adjusted so that the second joints of the children's fingers were at a right angle during the assessment and the children kept their arms by, but not against their sides. While standing, the children were asked to complete two trials of maximal effort with each hand. Grip strength was recorded to $0.5 \mathrm{~kg}$.

The timed plank provided a measure of muscular endurance. Instructions and data collection followed the protocol established by Boyer et al. [2]. Children were shown and asked to demonstrate a plank where their hips were in line with their head and feet and their feet were together. Once the child understood and could demonstrate the plank, he/she was asked to hold it as long as possible. The children were given one warning if their form failed and if/when a second warning was required the timing stopped. Time was recorded to the nearest tenth of a second.

Height was measured using a SECA (Hamburg, Germany) standiometer. Stretched stature was obtained for each child following CSEP protocol [4]. Children stood upright with shoes removed, feet together and heels and back in contact with the standiometer. Data collectors ensured the children's heads were in the Frankfort plane and lowered the headboard until it made firm contact. Height was recorded to the nearest tenth of a centimeter. A minimum of two trials was performed and if the difference between trials was greater than $0.5 \mathrm{~cm}$ a third trial was completed with the closest two averaged for the final height value.

Body mass was measured using a Taylor (Oak Brook, Illinois, USA) digital scale according to CSEP protocol [5]. Children stood on this scale without shoes and in light clothing. Two measurements were taken and recorded to the nearest tenth of a kilogram. A third trial was conducted if the first two measurements differed by more than $0.5 \mathrm{~kg}$. The two closest measurements were averaged to produce the final measurement of mass.

\subsection{Data Analysis}

Recognizing the geometric similarity in somatic growth and the impact of growth in area and volume, predictions can be made [1]. Strength is related to the cross-sectional area of muscle. Cardiorespiratory and muscular endurance are related to the volume of the heart, lungs, and vascular system. Dimensional analysis also involves the growth in height. As such, the height ratio was determined:

$$
\begin{aligned}
& \text { Height ratio } \\
& =\text { final measurement height }(\mathrm{cm}) \\
& \quad \div \text { initial height measurement }(\mathrm{cm}) .
\end{aligned}
$$

Then, the following formula was used to calculate the expected increases in right- and left-hand grip strength (using right-hand grip strength as the example):

$$
\begin{aligned}
& \text { Increase in right }- \text { hand grip strength expected } \\
& =\left(\begin{array}{l}
\text { height } \text { ratio }^{2} \mathrm{x} \text { initial right } \\
\text {-hand grip strength measurement }[\mathrm{kg}]
\end{array}\right) \\
& \text {-initial right }- \text { hand grip strength measurement }(\mathrm{kg})
\end{aligned}
$$

Note: since strength is related to the cross-sectional area of muscle, the height ratio was raised to the power of 2 .

The expected increases in the cardiorespiratory and muscular endurance measures (PACER and timed plank) were calculated as follows (with PACER used as the example):

$$
\begin{aligned}
& \text { Increase in PACER laps expected } \\
& =\left(\text { height } \text { ratio }^{3} \mathrm{x} \text { initial number of laps completed }\right) \\
& \quad \text {-initial number of laps completed }
\end{aligned}
$$

Note: Since, cardiorespiratory and muscular endurance are related to the volume of the heart, lungs and, blood, the height ratio was raised to the power of 3 .

The expected increases in performance for each physical competency measure (i.e., right- and left-hand grip, PACER, and timed plank) were then compared to the actual measurements found (time 2 - time 1). Four repeated-measures one tail t-tests were used to determine if there were significant differences between the expected and observed changes in physical competency measures across the two times points. Since multiple t-tests were used, the Bonferroni correction was applied to counteract the likelihood of finding significant differences due to multiple comparisons. By dividing the number of tests (i.e., 4$)$ by the alpha $(0.05)$, the more appropriate alpha for these tests was set at $\mathrm{p}<0.0125$. 


\section{Results}

Although 206 participants provided two data points, only $182(88.3 \%)$ had their height measured twice and could be included in the analyses. As presented in Table 1, of these 182 participants, 178 had two sets of data for the timed plank and right- and left-hand grip measures and 173 had two measures for the PACER. These data were further examined for outliers. Individuals with performances 2 standard deviations less than or greater than the mean were removed from the analyses. Final numbers included in the analyses are presented in Table 1.

Table 1. Number of participants with time 1 and 2 data; with and without outliers

\begin{tabular}{|c|c|c|}
\hline & $\begin{array}{c}\text { Participants with } \\
\text { two data points }\end{array}$ & $\begin{array}{c}\text { Participants with two data } \\
\text { points, outliers removed }\end{array}$ \\
\hline Timed plank & 178 & 173 \\
\hline PACER & 173 & 165 \\
\hline Left-hand grip & 178 & 168 \\
\hline Right-hand grip & 178 & 169 \\
\hline
\end{tabular}

Descriptive statistics are presented in Table 2 for the participants for both measurement periods (i.e., time 1 and time 2) for age, height, weight for the boys and girls separately. The data in Table 3 shows the boys' and girls' height measurements from time 1 and time 2 according to age at first measurement.

Expected increases in physical competency were calculated for the children's results in the left- and right-hand grip strength, plank, and PACER using the formulas previously presented. Actual changes in physical competencies were determined by subtracting the first measurement from the second measurement.

The data in Table 4 presents the average \pm standard deviation of the left- and right-hand grip strength, PACER, and timed plank scores from both measurement time points as well as the average \pm standard deviation for the expected and observed changes. The observed differences were significantly greater than expected for the left$\mathrm{t}(167)=-4.731, \mathrm{p}=0.000$, and right-hand grip strength; $\mathrm{t}(168)=-4.579, \mathrm{p}=0.000$. The observed changes in plank were significantly different than expected; $\mathrm{t}(172)=-2.743$, $\mathrm{p}=0.003$; however these differences were in a direction not expected, i.e., the average time for the plank decreased from time 1 to time 2. No significant differences were found between the expected and observed number of PACER laps between time 1 and time 2; $\mathrm{t}(164)=-1.023$, $\mathrm{p}=0.154$.

Table 2. Age, height, and weight (average \pm SD) for boys and girls for time 1 and 2

\begin{tabular}{|c|c|c|c|}
\hline \multicolumn{2}{|c|}{} & Time 1 & Time 2 \\
\hline \multirow{3}{*}{ Boys } & Age (yrs) & $9.96 \pm 0.69$ & $10.85 \pm 0.64$ \\
\cline { 2 - 4 } & Height (cm) & $140.97 \pm 8.42$ & $146.27 \pm 8.67$ \\
\cline { 2 - 4 } & Weight (kg) & $39.43 \pm 12.01$ & $43.24 \pm 13.59$ \\
\hline \multirow{3}{*}{ Girls } & Age (yrs) & $9.94 \pm 0.62$ & $10.94 \pm 0.57$ \\
\cline { 2 - 4 } & Height (cm) & $139.81 \pm 6.76$ & $145.76 \pm 7.08$ \\
\cline { 2 - 4 } & Weight (kg) & $36.96 \pm 8.40$ & $41.49 \pm 9.72$ \\
\hline
\end{tabular}

Table 3. Increases in height and weight (average \pm SD) for boys and girls from time 1 to time 2

\begin{tabular}{|c|c|c|c|}
\hline \multirow{4}{*}{ Boys } & Age (yrs) & Height (cm) & Weight (kg) \\
\cline { 2 - 4 } & 8 & $6.07 \pm 0.25$ & $3.67 \pm 0.52$ \\
\cline { 2 - 4 } & 9 & $4.91 \pm 1.71$ & $4.10 \pm 4.58$ \\
\cline { 2 - 4 } & 10 & $5.89 \pm 3.92$ & $3.03 \pm 4.35$ \\
\hline \multirow{3}{*}{ Girls } & 11 & $4.67 \pm 1.40$ & $3.40 \pm 2.59$ \\
\cline { 2 - 4 } & 9 & $6.11 \pm 2.69$ & $5.14 \pm 3.06$ \\
\cline { 2 - 4 } & 10 & $5.99 \pm 1.81$ & $3.78 \pm 2.90$ \\
\hline
\end{tabular}

Table 4. Physical competency measures (Average \pm SD): observed and expected results (Average \pm SD) for participants aged 8-12

\begin{tabular}{|c|c|c|c|c|}
\hline Physical Competency Measure & Time point & Physical competency measure score & Change expected & Change observed \\
\hline \multirow{2}{*}{ Left-hand grip (kg) } & 1 & $15.94 \pm 3.78$ & \multirow{2}{*}{$1.27 \pm 0.52$} & \multirow{2}{*}{$2.10 \pm 2.19$} \\
\hline & 2 & $18.05 \pm 4.22$ & & \\
\hline \multirow{2}{*}{ Right-hand grip (kg) } & 1 & $16.57 \pm 3.86$ & \multirow{2}{*}{$1.32 \pm 0.55$} & \multirow{2}{*}{$2.06 \pm 2.08$} \\
\hline & 2 & $18.63 \pm 4.29$ & & \\
\hline \multirow{2}{*}{ PACER (laps) } & 1 & $21.55 \pm 12.48$ & \multirow{2}{*}{$2.53 \pm 1.69$} & \multirow{2}{*}{$3.29 \pm 9.00$} \\
\hline & 2 & $24.83 \pm 13.95$ & & \\
\hline \multirow{2}{*}{ Timed plank (sec) } & 1 & $58.61 \pm 38.23$ & \multirow{2}{*}{$7.42 \pm 6.57$} & \multirow{2}{*}{$-0.62 \pm 35.10$} \\
\hline & 2 & $57.99 \pm 37.03$ & & \\
\hline
\end{tabular}

\section{Discussion}

The purpose of this study was to determine whether the physiologic changes associated with the normal growth of children explained the changes observed in physical competency measures obtained from children ages 8 to 11 years when first measured and 9 to 12 years at their second measurement. Results from the physical competency measures for strength (left- and right-hand grip), muscular endurance (timed plank) and cardiorespiratory endurance (PACER) are mixed. The performance in right- and lefthand grip strength was significantly greater than expected from growth in height. Although the observed and expected times for timed plank were significantly different, the average timed plank performance unexpectedly decreased over time. Finally, the lack of significant difference between the expected and observed PACER data suggested that the observed increase in performance 
(i.e., number of laps completed) could be explained by the children's growth in height from time 1 to time 2 .

An underlying assumption of dimensional analyses is that growth is geometrically similar. Dimensional analysis theorizes that calculations of expected increases in strength, muscular and cardiovascular endurance in various measures including grip strength, length of plank hold, and laps run in the PACER can be made using mathematical formulas that consider the cross-sectional area of the muscle or the volumetric area of the lungs [1]. As noted, the increases found in left- and right-hand grip strength were significantly greater than the expected values with an average difference of approximately $2 \mathrm{~kg}$. Similar results were found by Asmussen and HeebollNielsen [1] on 400 Danish school boys aged 7 to 16 years. These researchers [1] suggested raising the height ratio to the power of 3.274 would better predict expected increases in finger flexor strength.

Combined with the findings of the present study, one might conclude that other factors influence strength development in children besides geometric growth such as the maturation of the nervous system [1]. It is believed that by about 7 years of age approximately $95 \%$ of neural growth has been achieved [9]. Still, Housh et al. [6] suggested that as the human body reaches sexual maturity there is an increase in the number of neuromuscular junctions and nerves increase in myelination thus increasing the muscle's ability to perform work in the context of a muscular contraction. Therefore, there may be neural growth independent of geometric growth that increases the muscles ability to perform the skills of the physical capacity tests performed. It is difficult to make any estimation of maturation of the participants in this study, as no measurement was included for it, thus this idea could not be further examined. That said, a closer look at the average increases in height observed in this study of about 5-6 cm is indicative of growth in late childhood and not the more rapid rate of growth expected during puberty. It should also be pointed out that increases observed in grip strength may also be related to the participants' varying participation in daily physical activity, the types of activity participated in, and the change in participants' weight. Differences in grip strength related to types and amounts of physical activity participation could not be examined as this data was not collected.

As previously noted, a significant difference was found between expected and observed timed plank times, however, this observed difference was an average reduction of 0.62 seconds. This unexpected decrease in timed plank performance is contrary to what was expected in the normal growth of children. The psychobiologic model of exercise performance based on motivational intensity theory stipulates that performances in time to exhaustion tasks are governed by two factors: perceived exhaustion and potential motivation [10]. For the timed plank, participants were asked to perform the task until exhaustion. Exercising to exhaustion requires a great deal of motivation and understanding of the body and its capacities, particularly in children. External motivation may also play a role. Given there were two measurements, it is possible that participants who had not enjoyed their first experience may not have tried as hard the second time data were collected and stopped their second performance prior to exhaustion. Another explanation may relate to the different data collectors involved in collecting data as there may have been differences in the motivation provided to the participants.

The participants' increase in performance on the PACER at time 2 was explained by their growth in height. This finding was expected as the children were not engaged in any training or exercise programs intended to improve their cardiorespiratory endurance other than regular physical education classes. These results are worthy of noting and important to consider when various exercise or training programs are evaluated in that resultant increases in performance are partly due to increases in height and not just the exercise/training program.

A potential limitation to this study was the variability in data collectors for some of the physical competency measurements such as the timed plank and measures of grip strength. Although the data collectors attended mandatory training sessions including practical opportunities to collect data, there may have been discrepancies in the motivation provided to the participants in efforts to obtain maximal performance. Another limitation is the inability to account for maturational differences. That said, as previously noted the average growth in height for the girls and boys was about 5 to $6 \mathrm{~cm}$ over the year, and not the 8 to $12 \mathrm{~cm} /$ year as expected in pubertal growth [9]. These values are consistent with middle and late childhood growth in height. Strengths to this study include the sample size with repeat measurements on more than 173 children and use of standardized measurement tools and procedures to collect muscular strength, muscular endurance, and cardiorespiratory endurance. It should also be pointed out that the same individual collected the height and mass measures from the children and the same individual was in charge of the PACER protocol.

In conclusion, although the growth in height of these children accounted for some of the changes observed in the physical competency measures collected, it did not account for it all. Further, the impact of growth in height was inconsistent for the four measurements of physical competency analyzed in this study. As such, these results suggest other biologic and non-biologic factors influence the physical competencies of children. Future studies of repeated measures of physical competency in children should consider dimensional analyses as a means of controlling for the changes in performance expected because of the increase in size expected as part of the growth, maturation, and development of children.

\section{Acknowledgements}

The authors thank the children who participated in the CAPL research project and the data collectors involved.

\section{References}

[1] Asmussen, E. and Heebøll-Nielsen, K. A dimensional analysis of physical performance and growth in boys. Journal of Applied Physiology 7:593-603, 1955.

[2] Boyer, C., Tremblay, M.S., Saunders, T.J., McFarlane, A., Borghese, M., Lloyd, M., and Longmuir P.E. Feasibility, validity and reliability of the plank isometric hold as a field-based assessment of torso muscular endurance for children 8 to 12 years of age. Pediatric Exercise Science 25(3):407-422. 2013 
[3] Caroni, P., and Grandes, P. Nerve sprouting in innervated adult skeletal muscle induced by exposure to elevated levels of insulin-like growth factors. Journal of Cell Biology 110(4): 1307-1317. 1990.

[4] Canadian Society for Exercise Physiology (CSEP). Canadian Society for Exercise Physiology. Physical Activity Training for Health (CSEP-PATH). Ottawa, Ontario, Canada, 2013.

[5] Galganski, M.E., Fuglevand, A.J., and Enoka, R.M. Reduced control of motor output in a human hand muscle of elderly subjects during submaximal contractions. Journal of Neurophysiology 69(6):2108-2115.1993

[6] Harris, J.A., Jackson, C.M., Paterson, and D.G., Scammon, R.E. (Eds.). The measurement of man. University of Minnesota Press. 1930.

[7] Housh, T.J., Johnson, G.O., Housh, D.J., Stout, J.R., Weir, J.P., Weir, L.L., and Eckerson, J.M. Isokinetic peak torque in young wrestlers. Pediatric Exercise Science 8: 143-155. 1996.

[8] Lloyd, M., and Tremblay, M.S. Introducing the Canadian Assessment of Physical Literacy. In G. Baquet and S. Berthoin (Eds). Children and Exercise XXV: The Proceedings of the $25^{\text {th }}$ Pediatric Work Physiology Meeting, 2009.
[9] Lynch, N.A., Metter, E.J., Lindle, R.S., et al. Muscle quality: Ageassociated differences between arm and leg muscle groups. Journal of Applied Physiology 86(1):188-194. 1999.

[10] Malina, R.M., Bouchard, C., and Bar-Or, O. Growth, Maturation, and Physical Activity. 2nd edition. Human Kinetics, Champaign, IL, 2004.

[11] Marcora, S.M. Do we really need a central governor to explain brain regulation of exercise performance? European Journal of Applied Physiology 104(5):929-931. 2008.

[12] Meredith, M.D. and Welk, G.J. FitnessGram \& ActivityGram: Test Administration Manual. Dallas, Texas: The Cooper Institute, 2010.

[13] Rogol, A.D., Roemmich, J.N., and Clark, P.A. Growth at puberty. Journal of Adolescent Health 31(6):192-200. 2002.

[14] Scammon, R.E. The measurement of the body in childhood. In J.A., Harris, C.M. Jackson., D.G. Paterson, and R.E. Scammon, Eds). The Measurement of Man, Univ. of Minnesota Press, Minneapolis. 1930. 